\title{
Revised Chapman-Enskog analysis for a class of forcing schemes in the lattice Boltzmann method
}

\author{
Q. Li, ${ }^{*}$ P. Zhou, and H. J. Yan \\ School of Energy Science and Engineering, Central South University, Changsha 410083, China \\ (Received 8 May 2016; revised manuscript received 2 September 2016; published 21 October 2016)
}

\begin{abstract}
In the lattice Boltzmann (LB) method, the forcing scheme, which is used to incorporate an external or internal force into the LB equation, plays an important role. It determines whether the force of the system is correctly implemented in an LB model and affects the numerical accuracy. In this paper we aim to clarify a critical issue about the Chapman-Enskog analysis for a class of forcing schemes in the LB method in which the velocity in the equilibrium density distribution function is given by $\mathbf{u}=\sum_{\alpha} \mathbf{e}_{\alpha} f_{\alpha} / \rho$, while the actual fluid velocity is defined as $\hat{\mathbf{u}}=\mathbf{u}+\delta_{t} \mathbf{F} /(2 \rho)$. It is shown that the usual Chapman-Enskog analysis for this class of forcing schemes should be revised so as to derive the actual macroscopic equations recovered from these forcing schemes. Three forcing schemes belonging to the above class are analyzed, among which Wagner's forcing scheme [A. J. Wagner, Phys. Rev. E 74, 056703 (2006)] is shown to be capable of reproducing the correct macroscopic equations. The theoretical analyses are examined and demonstrated with two numerical tests, including the simulation of Womersley flow and the modeling of flat and circular interfaces by the pseudopotential multiphase LB model.
\end{abstract}

DOI: 10.1103/PhysRevE.94.043313

\section{INTRODUCTION}

The lattice Boltzmann (LB) method, which is a special discrete solver for mimicking the kinetic Boltzmann equation, has been developed into an efficient mesoscopic numerical approach for simulating fluid flow and heat transfer [1,2]. Owing to its kinetic features, the LB method has attracted significant attention in the past two decades and has been applied in a large variety of fields [3-5]. In many systems, an external or internal force is involved. For these systems, a forcing scheme is needed to incorporate the force into the LB equation. In the literature, various forcing schemes have been developed, such as the forcing schemes proposed by Shan and Chen [6,7], He et al. [8], Buick and Greated [9], Ladd and Verberg [10], and Guo et al. [11]. To date, it has been generally accepted that the forcing scheme of Guo et al. is able to recover the correct unsteady macroscopic equations at the Navier-Stokes level.

Moreover, in the study of Guo et al. [11] a theoretical framework is given for deriving the macroscopic equations recovered from the forcing schemes that can be expanded in a power series in the lattice velocity $\mathbf{e}_{\alpha}$. Some of the previous forcing schemes have been analyzed in Ref. [11]. After the study of Guo et al., several alternative forcing schemes were developed. For example, Wagner [12] proposed a forcing scheme by conducting a Taylor expansion analysis for the LB equation and Kupershtokh et al. [13] presented a forcing scheme based on the work of He et al. [8]. It can be found that these two forcing schemes share the following feature: The velocity in the equilibrium density distribution function is given by $\mathbf{u}=\sum_{\alpha} \mathbf{e}_{\alpha} f_{\alpha} / \rho$, while the actual fluid velocity is defined as $\hat{\mathbf{u}}=\mathbf{u}+\delta_{t} \mathbf{F} /(2 \rho)$, where $\mathbf{F}$ is the force and $\delta_{t}$ is

\footnotetext{
*Corresponding author: qingli@csu.edu.cn
}

Published by the American Physical Society under the terms of the Creative Commons Attribution 3.0 License. Further distribution of this work must maintain attribution to the author(s) and the published article's title, journal citation, and DOI. the time step. In fact, some previous forcing schemes such as a forcing scheme proposed by Ladd and Verberg [10], which was analyzed in Ref. [11], also belong to this class of forcing schemes.

According to the available Chapman-Enskog analysis in the literature (e.g., Refs. [9-11]), for this class of forcing schemes, it can be found that Wagner's forcing scheme cannot recover the correct Navier-Stokes equations. However, Wagner showed [12] that his forcing scheme was obtained based on the Taylor expansion analysis for recovering the correct macroscopic equations. To clarify such an issue, in the present study we demonstrate that the usual Chapman-Enskog analysis in the literature for the aforementioned class of forcing schemes should be modified in order to derive the actual macroscopic equations recovered from these forcing schemes.

The revised Chapman-Enskog analysis shows that Wagner's forcing scheme can correctly recover the macroscopic equations at the Navier-Stokes level, confirming the consistency between the Chapman-Enskog analysis and the Taylor expansion analysis. Besides Wagner's forcing scheme, another two forcing schemes are also analyzed. Two numerical tests are employed to validate the theoretical analysis. The rest of the present paper is organized as follows. The forcing schemes are introduced in Sec. II. The theoretical and numerical analyses are presented in Secs. III and IV, respectively. Finally, a brief summary is given in Sec. V.

\section{FORCING SCHEMES}

For the aforementioned class of forcing schemes, the LB equation is given as follows $[10,12,13]$ :

$f_{\alpha}\left(\mathbf{x}+\mathbf{e}_{\alpha} \delta_{t}, t+\delta_{t}\right)-f_{\alpha}(\mathbf{x}, t)=-\frac{1}{\tau}\left[f_{\alpha}-f_{\alpha}^{\mathrm{eq}}(\rho, \mathbf{u})\right]+F_{\alpha}$,

where $f_{\alpha}$ is the density distribution function, $f_{\alpha}^{\text {eq }}$ is the equilibrium density distribution function, $\tau$ is the nondimensional relaxation time, and $F_{\alpha}$ is the forcing term. Using the D2Q9 lattice model, the equilibrium distribution function can be 
given by

$$
f_{\alpha}^{\mathrm{eq}}(\rho, \mathbf{u})=\omega_{\alpha} \rho\left[1+\frac{\mathbf{e}_{\alpha} \cdot \mathbf{u}}{c_{s}^{2}}+\frac{\mathbf{u u}:\left(\mathbf{e}_{\alpha} \mathbf{e}_{\alpha}-c_{s}^{2} \mathbf{I}\right)}{2 c_{s}^{4}}\right],
$$

where the weights $\omega_{\alpha}$ are $\omega_{0}=4 / 9, \omega_{1-4}=1 / 9$, and $\omega_{5-8}=$ $1 / 36$. The velocity $\mathbf{u}$ and the actual fluid velocity $\hat{\mathbf{u}}$ are given by, respectively,

$$
\rho \mathbf{u}=\sum_{\alpha} \mathbf{e}_{\alpha} f_{\alpha}, \quad \rho \hat{\mathbf{u}}=\rho \mathbf{u}+\frac{\delta_{t}}{2} \mathbf{F} .
$$

The forcing schemes proposed by Wagner [12], Kupershtokh et al. [13], and Ladd and Verberg [10] fall into the above category, although the forcing terms $F_{\alpha}$ of these schemes are different. By conducting the Taylor expansion analysis of Eq. (1), Wagner [12] pointed out that the forcing term in Eq. (1) should satisfy the following constraints [see Eqs. (19)-(21) and (59) in Ref. [12]]:

$$
\begin{gathered}
\sum_{\alpha} F_{\alpha, \mathrm{Wa}}=0, \quad \sum_{\alpha} \mathbf{e}_{\alpha} F_{\alpha, \mathrm{Wa}}=\delta_{t} \mathbf{F}, \\
\sum_{\alpha} \mathbf{e}_{\alpha} \mathbf{e}_{\alpha} F_{\alpha, \mathrm{Wa}}=\delta_{t}\left[(\mathbf{u F}+\mathbf{F u})+\delta_{t}\left(1-\frac{1}{4 \tau}\right) \frac{\mathbf{F F}}{\rho}\right] .
\end{gathered}
$$

Here $F_{\alpha, \text { Wa }}$ represents the forcing term of Wagner's forcing scheme. Note that a higher-order term has been neglected in Eq. (5).

Kupershtokh et al. [13] presented a forcing scheme, namely, the so-called exact-difference-method forcing scheme, that obeys Eqs. (1)-(3) with the following forcing term:

$$
F_{\alpha, \text { EDM }}=f_{\alpha}^{\mathrm{eq}}(\rho, \mathbf{u}+\Delta \mathbf{u})-f_{\alpha}^{\mathrm{eq}}(\rho, \mathbf{u}),
$$

where $\Delta \mathbf{u}=\mathbf{F} \delta_{t} / \rho$. Substituting the expression of $f_{\alpha}^{\text {eq }}$ into Eq. (6), it can be found that $F_{\alpha, \text { EDM }}$ is identical to [14]

$$
F_{\alpha, \mathrm{EDM}}=\omega_{\alpha} \delta_{t}\left[\frac{\mathbf{F} \cdot \mathbf{e}_{\alpha}}{c_{s}^{2}}+\frac{\left(\mathbf{v}_{\mathrm{EDM}} \mathbf{F}+\mathbf{F} \mathbf{v}_{\mathrm{EDM}}\right):\left(\mathbf{e}_{\alpha} \mathbf{e}_{\alpha}-c_{s}^{2} \mathbf{I}\right)}{2 c_{s}^{4}}\right],
$$

where $\mathbf{v}_{\mathrm{EDM}}=\mathbf{u}+\Delta \mathbf{u} / 2=\mathbf{u}+\delta_{t} \mathbf{F} /(2 \rho)$. Evaluating the moments of $F_{\alpha, \text { EDM }}$, we can obtain

$$
\begin{gathered}
\sum_{\alpha} F_{\alpha, \mathrm{EDM}}=0, \quad \sum_{\alpha} \mathbf{e}_{\alpha} F_{\alpha, \mathrm{EDM}}=\delta_{t} \mathbf{F}, \\
\sum_{\alpha} \mathbf{e}_{\alpha} \mathbf{e}_{\alpha} F_{\alpha, \mathrm{EDM}}=\delta_{t}\left((\mathbf{u F}+\mathbf{F u})+\delta_{t} \frac{\mathbf{F F}}{\rho}\right) .
\end{gathered}
$$

Comparing Eq. (9) with Eq. (5), we can see that the second-order moments of the forcing terms of Wagner and Kupershtokh et al. are almost the same except that the coefficients in front of the term $\mathbf{F F} / \rho$ are different. Similarly, it can be found that Ladd and Verberg's forcing term yields the following moments [see Eq. (28) and Sec. 5.3 in Ref. [10]]:

$$
\begin{gathered}
\sum_{\alpha} F_{\alpha, \text { Ladd }}=0, \quad \sum_{\alpha} \mathbf{e}_{\alpha} F_{\alpha, \text { Ladd }}=\delta_{t} \mathbf{F}, \\
\sum_{\alpha} \mathbf{e}_{\alpha} \mathbf{e}_{\alpha} F_{\alpha, \text { Ladd }}=\delta_{t}(\mathbf{u F}+\mathbf{F u}) .
\end{gathered}
$$

Unlike Eqs. (5) and (9), Eq. (11) does not contain the term related to $\mathbf{F F} / \rho$.

For comparison, the forcing scheme of Guo et al. is also given here, which consists of the following LB equation [11]:

$$
\begin{aligned}
& f_{\alpha}\left(\mathbf{x}+\mathbf{e}_{\alpha} \delta_{t}, t+\delta_{t}\right)-f_{\alpha}(\mathbf{x}, t) \\
& \quad=-\frac{1}{\tau}\left[f_{\alpha}-f_{\alpha}^{\mathrm{eq}}(\rho, \hat{\mathbf{u}})\right]+F_{\alpha, \mathrm{Guo}},
\end{aligned}
$$

with the forcing term

$$
\begin{aligned}
& F_{\alpha, \text { Guo }} \\
& \quad=\omega_{\alpha}\left(1-\frac{1}{2 \tau}\right) \delta_{t}\left[\frac{\mathbf{F} \cdot \mathbf{e}_{\alpha}}{c_{s}^{2}}+\frac{(\hat{\mathbf{u}} \mathbf{F}+\mathbf{F} \hat{\mathbf{u}}):\left(\mathbf{e}_{\alpha} \mathbf{e}_{\alpha}-c_{s}^{2} \mathbf{I}\right)}{2 c_{s}^{4}}\right],
\end{aligned}
$$

which leads to the following moments:

$$
\begin{gathered}
\sum_{\alpha} F_{\alpha, \mathrm{Guo}}=0, \quad \sum_{\alpha} \mathbf{e}_{\alpha} F_{\alpha, \mathrm{Guo}}=\delta_{t}\left(1-\frac{1}{2 \tau}\right) \mathbf{F}, \\
\sum_{\alpha} \mathbf{e}_{\alpha} \mathbf{e}_{\alpha} F_{\alpha, \mathrm{Guo}}=\delta_{t}\left(1-\frac{1}{2 \tau}\right)(\hat{\mathbf{u}} \mathbf{F}+\mathbf{F} \hat{\mathbf{u}}) .
\end{gathered}
$$

For the forcing scheme of Guo et al., the actual fluid velocity and the velocity in the equilibrium density distribution function $f_{\alpha}^{\text {eq }}(\rho, \hat{\mathbf{u}})$ and the forcing term $F_{\alpha, \text { Guo }}$ are all given by $\hat{\mathbf{u}}=$ $\mathbf{u}+\delta_{t} \mathbf{F} /(2 \rho)$, with $\mathbf{u}=\sum_{\alpha} \mathbf{e}_{\alpha} f_{\alpha} / \rho$.

\section{THEORETICAL ANALYSIS}

\section{A. Multiscale expansions}

The Chapman-Enskog analysis has been widely utilized in the LB community to derive the macroscopic equations recovered from LB models. To perform such an analysis, the following multiscale expansions are usually introduced [9]:

$$
\begin{gathered}
f_{\alpha}=f_{\alpha}^{(0)}+\varepsilon f_{\alpha}^{(1)}+\varepsilon^{2} f_{\alpha}^{(2)}+\cdots, \\
\partial_{t}=\varepsilon \partial_{t 1}+\varepsilon^{2} \partial_{t 2}, \quad \nabla=\varepsilon \nabla_{1}, \quad \mathbf{F}=\varepsilon \mathbf{F}_{1},
\end{gathered}
$$

where $\varepsilon$ is the expansion parameter. According to the expansion of the force $\mathbf{F}$, the forcing term is also expanded as $F_{\alpha}=\varepsilon F_{1 \alpha}$. Using these expansions, the LB equation with a forcing term can be written in the consecutive order of $\varepsilon$,

$$
\begin{gathered}
O\left(\varepsilon^{0}\right): f_{\alpha}^{(0)}=f_{\alpha}^{\mathrm{eq}} \\
O\left(\varepsilon^{1}\right):\left(\partial_{t 1}+\mathbf{e}_{\alpha} \cdot \nabla_{1}\right) f_{\alpha}^{(0)}=-\frac{1}{\tau \delta_{t}} f_{\alpha}^{(1)}+\frac{1}{\delta_{t}} F_{1 \alpha} \\
O\left(\varepsilon^{2}\right): \partial_{t 2} f_{\alpha}^{(0)}+\left(\partial_{t 1}+\mathbf{e}_{\alpha} \cdot \nabla_{1}\right) f_{\alpha}^{(1)}+\frac{\delta_{t}}{2}\left(\partial_{t 1}+\mathbf{e}_{\alpha} \cdot \nabla_{1}\right)^{2} f_{\alpha}^{(0)} \\
=-\frac{1}{\tau \delta_{t}} f_{\alpha}^{(2)}
\end{gathered}
$$

for $\mathrm{O}\left(\varepsilon^{0}\right), \mathrm{O}\left(\varepsilon^{1}\right)$, and $\mathrm{O}\left(\varepsilon^{2}\right)$, respectively. With the aid of Eq. (19), Eq. (20) can be rewritten as

$$
\begin{aligned}
& \partial_{t 2} f_{\alpha}^{(0)}+\left(\partial_{t 1}+\mathbf{e}_{\alpha} \cdot \nabla_{1}\right)\left(1-\frac{1}{2 \tau}\right) f_{\alpha}^{(1)}+\frac{1}{2}\left(\partial_{t 1}+\mathbf{e}_{\alpha} \cdot \nabla_{1}\right) F_{1 \alpha} \\
& \quad=-\frac{1}{\tau \delta_{t}} f_{\alpha}^{(2)} .
\end{aligned}
$$


In the literature [10,11], the above equations were applied to both Eqs. (1) and (12) by setting $f_{\alpha}^{\text {eq }}$ in Eq. (18) to $f_{\alpha}^{\text {eq }}(\rho, \mathbf{u})$ and $f_{\alpha}^{\mathrm{eq}}(\rho, \hat{\mathbf{u}})$, respectively.

\section{B. Original analysis}

Here the original analysis denotes the usual ChapmanEnskog analysis of Eq. (1), in which $f_{\alpha}^{\text {eq }}$ in Eq. (18) is taken as $f_{\alpha}^{\text {eq }}(\rho, \mathbf{u})$, which leads to $\sum_{\alpha} \mathbf{e}_{\alpha} f_{\alpha}^{(n)}=0(n \neq 0)$ since $\rho \mathbf{u}=\sum_{\alpha} \mathbf{e}_{\alpha} f_{\alpha}^{\text {eq }}=\sum_{\alpha} \mathbf{e}_{\alpha} f_{\alpha}$. Meanwhile, it can be found that $\sum_{\alpha} f_{\alpha}^{(n)}=0(n \neq 0)$. With these results, the following equations can be obtained by taking the summations of Eqs. (19) and (21), respectively,

$$
\begin{gathered}
\partial_{t 1} \rho+\nabla_{1} \cdot(\rho \mathbf{u})=0, \\
\partial_{t 2} \rho+\frac{1}{2} \nabla_{1} \cdot\left(\delta_{t} \mathbf{F}_{1}\right)=0 .
\end{gathered}
$$

Combining Eq. (22) with Eq. (23) using the relationships in Eq. (17), we have

$$
\partial_{t} \rho+\nabla \cdot(\rho \hat{\mathbf{u}})=0
$$

where $\hat{\mathbf{u}}=\mathbf{u}+\delta_{t} \mathbf{F} /(2 \rho)$ is the actual fluid velocity. The above equation is the continuity equation. Similarly, the first-order moments of Eqs. (19) and (21) yield, respectively,

$$
\begin{gathered}
\partial_{t 1}(\rho \mathbf{u})+\nabla_{1} \cdot(\rho \mathbf{u u})=-\nabla_{1} p+\mathbf{F}_{1}, \\
\partial_{t 2}(\rho \mathbf{u})+\nabla_{1} \cdot\left[\left(1-\frac{1}{2 \tau}\right) \sum_{\alpha} \mathbf{e}_{\alpha} \mathbf{e}_{\alpha} f_{\alpha}^{(1)}\right] \\
+\frac{\delta_{t}}{2} \partial_{t 1} \mathbf{F}_{1}+\frac{1}{2} \nabla_{1} \cdot \sum_{\alpha} \mathbf{e}_{\alpha} \mathbf{e}_{\alpha} F_{1 \alpha}=0,
\end{gathered}
$$

where $p=\rho c_{s}^{2}$. In addition, from Eq. (19) the following equation can be obtained:

$$
\begin{aligned}
\sum_{\alpha} \mathbf{e}_{\alpha} \mathbf{e}_{\alpha} f_{\alpha}^{(1)}= & -\tau\left[\delta_{t} \partial_{t 1}\left(\sum_{\alpha} \mathbf{e}_{\alpha} \mathbf{e}_{\alpha} f_{\alpha}^{(0)}\right)+\delta_{t} \nabla_{1}\right. \\
& \left.\cdot\left(\sum_{\alpha} \mathbf{e}_{\alpha} \mathbf{e}_{\alpha} \mathbf{e}_{\alpha} f_{\alpha}^{(0)}\right)-\sum_{\alpha} \mathbf{e}_{\alpha} \mathbf{e}_{\alpha} F_{1 \alpha}\right] .
\end{aligned}
$$

Substituting Eq. (27) into Eq. (26) gives

$$
\partial_{t 2}(\rho \mathbf{u})+\nabla_{1} \cdot \Pi^{(1)}+\frac{\delta_{t}}{2} \partial_{t 1} \mathbf{F}_{1}+\nabla_{1} \cdot \tau \sum_{\alpha} \mathbf{e}_{\alpha} \mathbf{e}_{\alpha} F_{1 \alpha}=0,
$$

where $\Pi^{(1)}$ is given by

$$
\begin{aligned}
\boldsymbol{\Pi}^{(1)}= & -\left(\tau-\frac{1}{2}\right) \delta_{t}\left[\partial_{t 1}\left(\sum_{\alpha} \mathbf{e}_{\alpha} \mathbf{e}_{\alpha} f_{\alpha}^{(0)}\right)\right. \\
& \left.+\nabla_{1} \cdot\left(\sum_{\alpha} \mathbf{e}_{\alpha} \mathbf{e}_{\alpha} \mathbf{e}_{\alpha} f_{\alpha}^{(0)}\right)\right] .
\end{aligned}
$$

According to the treatment $f_{\alpha}^{(0)}=f_{\alpha}^{\mathrm{eq}}(\rho, \mathbf{u})$ as well as Eqs. (22) and (25), the following expression can be obtained:

$$
\boldsymbol{\Pi}^{(1)}=-\left(\tau-\frac{1}{2}\right) \delta_{t}\left\{\rho c_{s}^{2}\left[\nabla_{1} \mathbf{u}+\left(\nabla_{1} \mathbf{u}\right)^{\mathrm{T}}\right]+\mathbf{u} \mathbf{F}_{1}+\mathbf{F}_{1} \mathbf{u}\right\} .
$$

Substituting Eq. (30) into Eq. (28) and then combining the result with Eq. (25), we can obtain

$$
\partial_{t}(\rho \mathbf{u})+\nabla \cdot(\rho \mathbf{u u})=-\nabla p+\nabla \cdot\left\{\rho v\left[\nabla \mathbf{u}+(\nabla \mathbf{u})^{\mathrm{T}}\right]\right\}+\mathbf{F}-\frac{\delta_{t}}{2} \varepsilon \partial_{t 1} \mathbf{F}+\nabla \cdot\left[\left(\tau-\frac{1}{2}\right) \delta_{t}(\mathbf{u F}+\mathbf{F u})-\tau \sum_{\alpha} \mathbf{e}_{\alpha} \mathbf{e}_{\alpha} F_{\alpha}\right],
$$

where $v=(\tau-0.5) c_{s}^{2} \delta_{t}$ is the kinematic viscosity. The final form of Eq. (31) depends on the second-order moment of the forcing term, namely, $\sum_{\alpha} \mathbf{e}_{\alpha} \mathbf{e}_{\alpha} F_{\alpha}$. For example, if $\sum_{\alpha} \mathbf{e}_{\alpha} \mathbf{e}_{\alpha} F_{\alpha}=0$, we can obtain Eq. (21b) in Ref. [11].

In the above-mentioned class of forcing schemes, the actual fluid velocity is defined as $\hat{\mathbf{u}}=\mathbf{u}+\delta_{t} \mathbf{F} / 2 \rho$. Hence a transformation can be made from Eq. (31) to

$$
\begin{aligned}
\partial_{t}(\rho \hat{\mathbf{u}})+\nabla \cdot(\rho \hat{\mathbf{u}} \mathbf{u})= & -\nabla p+\nabla \cdot\left\{\rho v\left[\nabla \hat{\mathbf{u}}+(\nabla \hat{\mathbf{u}})^{\mathrm{T}}\right]\right\}+\mathbf{F}-\nabla \cdot\left\{\rho v\left[\nabla \mathbf{a}+(\nabla \mathbf{a})^{\mathrm{T}}\right]\right\}+\frac{\delta_{t}}{2} \varepsilon^{2} \partial_{t 2} \mathbf{F} \\
& +\nabla \cdot\left[\tau \delta_{t}(\mathbf{u} \mathbf{F}+\mathbf{F u})+\delta_{t}^{2} \frac{\mathbf{F F}}{4 \rho}-\tau \sum_{\alpha} \mathbf{e}_{\alpha} \mathbf{e}_{\alpha} F_{\alpha}\right],
\end{aligned}
$$

where $\mathbf{a}=\hat{\mathbf{u}}-\mathbf{u}=\delta_{t} \mathbf{F} / 2 \rho$. The following relationships have been utilized in deriving Eq. (32):

$$
\begin{aligned}
\partial_{t} \mathbf{F} & =\varepsilon \partial_{t 1} \mathbf{F}+\varepsilon^{2} \partial_{t 2} \mathbf{F}, \\
\rho \hat{\mathbf{u}} \mathbf{u} & =\rho \mathbf{u u}+\frac{\delta_{t}}{2}(\mathbf{u F}+\mathbf{F u})+\delta_{t}^{2} \frac{\mathbf{F F}}{4 \rho} .
\end{aligned}
$$

The left-hand side of Eq. (32) together with the first three terms on the right-hand side of Eq. (32) constitutes the correct macroscopic momentum equation and the remaining terms are regarded as error terms. For Wagner's forcing scheme, the following error terms would be obtained according to Eqs. (5) and (32):

$$
\begin{aligned}
\mathbf{E r}_{\mathrm{Wa}}= & \frac{\delta_{t}}{2} \varepsilon^{2} \partial_{t 2} \mathbf{F}-\nabla \cdot\left\{\rho v\left[\nabla \mathbf{a}+(\nabla \mathbf{a})^{\mathrm{T}}\right]\right\} \\
& +\nabla \cdot\left[\delta_{t}^{2}\left(\frac{1}{2}-\tau\right) \frac{\mathbf{F F}}{\rho}\right] .
\end{aligned}
$$

Similarly, for the forcing schemes of Kupershtokh et al. and Ladd and Verberg, the following error terms are obtained, 
respectively:

$$
\begin{aligned}
\mathbf{E r}_{\mathrm{EDM}}= & \frac{\delta_{t}}{2} \varepsilon^{2} \partial_{t 2} \mathbf{F}-\nabla \cdot\left\{\rho v\left[\nabla \mathbf{a}+(\nabla \mathbf{a})^{\mathrm{T}}\right]\right\} \\
& +\delta_{t}^{2} \nabla \cdot\left[\left(\frac{1}{4}-\tau\right) \frac{\mathbf{F F}}{\rho}\right], \\
\mathbf{E r}_{\text {Ladd }}= & \frac{\delta_{t}}{2} \varepsilon^{2} \partial_{t 2} \mathbf{F}-\nabla \cdot\left\{\rho v\left[\nabla \mathbf{a}+(\nabla \mathbf{a})^{\mathrm{T}}\right]\right\} \\
& +\delta_{t}^{2} \nabla \cdot\left(\frac{\mathbf{F F}}{4 \rho}\right) .
\end{aligned}
$$

According to the above analysis, there are many error terms in the macroscopic momentum equations recovered from the forcing schemes proposed by Wagner [12], Kupershtokh et al. [13], and Ladd and Verberg [10]. In other words, the above analysis tells us that all of these forcing schemes cannot recover the correct macroscopic momentum equation at the Navier-Stokes level. However, as mentioned in the Introduction, Wagner's forcing scheme was constructed based on the Taylor expansion analysis for recovering the correct macroscopic equations. According to Wagner's work [12], the following constraint should be satisfied so as to recover the momentum equation at the Navier-Stokes level [see Eq. (59) in the reference and neglect a high-order term]:

$$
\tau \Psi=\delta_{t}\left(\tau-\frac{1}{4}\right) \frac{\mathbf{F F}}{\rho},
$$

where $\boldsymbol{\Psi}=\sum_{\alpha}\left(\mathbf{e}_{\alpha}-\mathbf{u}\right)\left(\mathbf{e}_{\alpha}-\mathbf{u}\right) F_{\alpha} \equiv \sum_{\alpha} \mathbf{e}_{\alpha} \mathbf{e}_{\alpha} F_{\alpha}-\delta_{t}(\mathbf{u F}+$ $\mathbf{F u}$ ). It can be verified that Eq. (37) is satisfied when $\sum_{\alpha} \mathbf{e}_{\alpha} \mathbf{e}_{\alpha} F_{\alpha}$ is defined by Eq. (5). Therefore, the question is raised of whether the Taylor expansion analysis is inconsistent with the Chapman-Enskog analysis even at the Navier-Stokes level.

\section{Revised analysis}

To clarify the above inconsistency, we would like to introduce an effective forcing term for Wagner's forcing scheme. Since the actual fluid velocity is defined as $\hat{\mathbf{u}}=\mathbf{u}+\delta_{t} \mathbf{F} /(2 \rho)$, it is natural to rewrite $f_{\alpha}^{\text {eq }}(\rho, \mathbf{u})$ in Eq. (1) as follows:

$$
f_{\alpha}^{\mathrm{eq}}(\rho, \mathbf{u}) \equiv f_{\alpha}^{\mathrm{eq}}(\rho, \hat{\mathbf{u}})-\left[f_{\alpha}^{\mathrm{eq}}(\rho, \hat{\mathbf{u}})-f_{\alpha}^{\mathrm{eq}}(\rho, \mathbf{u})\right] .
$$

Correspondingly, Eq. (1) can be rewritten as

$$
f_{\alpha}\left(\mathbf{x}+\mathbf{e}_{\alpha} \delta_{t}, t+\delta_{t}\right)-f_{\alpha}(\mathbf{x}, t)=-\frac{f_{\alpha}-f_{\alpha}^{\mathrm{eq}}(\rho, \hat{\mathbf{u}})}{\tau}+\widehat{F}_{\alpha},
$$

where the effective forcing term $\widehat{F}_{\alpha}$ is given by

$$
\widehat{F}_{\alpha}=F_{\alpha}-\frac{f_{\alpha}^{\mathrm{eq}}(\rho, \hat{\mathbf{u}})-f_{\alpha}^{\mathrm{eq}}(\rho, \mathbf{u})}{\tau} .
$$

It can be seen that Eq. (39) takes the same form as Eq. (12), although the forcing term may be different.

For Wagner's forcing scheme, the following moments of the effective forcing term $\widehat{F}_{\alpha, \text { Wa }}$ can be obtained from Eqs. (4), (5), and (40) with the relationship $\hat{\mathbf{u}}=\mathbf{u}+\delta_{t} \mathbf{F} /(2 \rho)$ :

$$
\begin{aligned}
\sum_{\alpha} \widehat{F}_{\alpha, \mathrm{Wa}} & =0, \quad \sum_{\alpha} \mathbf{e}_{\alpha} \widehat{F}_{\alpha, \mathrm{Wa}}=\delta_{t}\left(1-\frac{1}{2 \tau}\right) \mathbf{F}, \\
\sum_{\alpha} \mathbf{e}_{\alpha} \mathbf{e}_{\alpha} \widehat{F}_{\alpha, \mathrm{Wa}} & =\delta_{t}\left(1-\frac{1}{2 \tau}\right)\left[(\mathbf{u F}+\mathbf{F u})+\delta_{t} \frac{\mathbf{F F}}{\rho}\right] .
\end{aligned}
$$

Since $(\mathbf{u F}+\mathbf{F u})+\delta_{t} \mathbf{F F} / \rho \equiv(\hat{\mathbf{u} F}+\mathbf{F} \hat{\mathbf{u}})$, it can be found that Eqs. (41) and (42) are identical to the results of the forcing scheme of Guo et al., namely, Eqs. (14) and (15), respectively. Actually, according to Eqs. (4) and (5), the original forcing term of Wagner's forcing scheme can be defined as

$$
\begin{aligned}
F_{\alpha, \text { Wa }}= & \omega_{\alpha} \delta_{t}\left\{\frac{\mathbf{F} \cdot \mathbf{e}_{\alpha}}{c_{s}^{2}}+\left[(\mathbf{u F}+\mathbf{F u})+\delta_{t}\left(1-\frac{1}{4 \tau}\right) \frac{\mathbf{F F}}{\rho}\right]\right. \\
& \left.: \frac{\left(\mathbf{e}_{\alpha} \mathbf{e}_{\alpha}-c_{s}^{2} \mathbf{I}\right)}{2 c_{s}^{4}}\right\} .
\end{aligned}
$$

Meanwhile, with the aid of Eq. (2), we can obtain

$$
\begin{aligned}
\frac{f_{\alpha}^{\mathrm{eq}}(\rho, \hat{\mathbf{u}})-f_{\alpha}^{\mathrm{eq}}(\rho, \mathbf{u})}{\tau} & \\
= & \frac{\rho \omega_{\alpha}}{\tau}\left[\frac{\mathbf{a} \cdot \mathbf{e}_{\alpha}}{c_{s}^{2}}+\frac{(\mathbf{u a}+\mathbf{a u}+\mathbf{a a}):\left(\mathbf{e}_{\alpha} \mathbf{e}_{\alpha}-c_{s}^{2} \mathbf{I}\right)}{2 c_{s}^{4}}\right] \\
= & \omega_{\alpha} \delta_{t}\left[\frac{1}{2 \tau} \frac{\mathbf{F} \cdot \mathbf{e}_{\alpha}}{c_{s}^{2}}+\left(\frac{1}{2 \tau}(\mathbf{u F}+\mathbf{F u})+\frac{\delta_{t}}{4 \tau} \frac{\mathbf{F F}}{\rho}\right)\right. \\
& \left.: \frac{\left(\mathbf{e}_{\alpha} \mathbf{e}_{\alpha}-c_{s}^{2} \mathbf{I}\right)}{2 c_{s}^{4}}\right],
\end{aligned}
$$

where $\mathbf{a}=\hat{\mathbf{u}}-\mathbf{u}=\delta_{t} \mathbf{F} / 2 \rho$. Then the following effective forcing term can be established for Wagner's forcing scheme by substituting Eqs. (43) and (44) into Eq. (40):

$$
\begin{aligned}
\widehat{F}_{\alpha, \text { Wa }}= & \omega_{\alpha}\left(1-\frac{1}{2 \tau}\right) \delta_{t}\left[\frac{\mathbf{F} \cdot \mathbf{e}_{\alpha}}{c_{s}^{2}}+\left(\mathbf{u F}+\mathbf{F u}+\delta_{t} \frac{\mathbf{F F}}{\rho}\right)\right. \\
& \left.: \frac{\left(\mathbf{e}_{\alpha} \mathbf{e}_{\alpha}-c_{s}^{2} \mathbf{I}\right)}{2 c_{s}^{4}}\right] .
\end{aligned}
$$

Using the relationship below Eq. (42), it can be readily verified that the above forcing term is just the forcing term of Guo et al. defined by Eq. (13). Based on Eqs. (39) and (45), we can conclude that Wagner's forcing scheme is theoretically identical to the forcing scheme of Guo et al. at the Navier-Stokes level. In other words, these two forcing schemes are theoretically equivalent, although they are constructed according to the Taylor expansion analysis and the ChapmanEnskog analysis, respectively.

Now it is clear that the so-called inconsistency mentioned in the preceding section is attributed to the problem that $f_{\alpha}^{(0)}$ in Eq. (18) is treated as $f_{\alpha}^{(0)}=f_{\alpha}^{\text {eq }}(\rho, \mathbf{u})$ in the usual Chapman-Enskog analysis for the aforementioned class of forcing schemes. According to the above analysis, a direct treatment for deriving the actual macroscopic equations from these forcing schemes is to utilize the transformation given by Eqs. (38) and (39). For such a treatment, Eqs. (18) and (19) together with Eq. (21) are still applicable. However, $f_{\alpha}^{\mathrm{eq}}$ in Eq. (18) should be taken as $f_{\alpha}^{\text {eq }}(\rho, \hat{\mathbf{u}})$. Meanwhile, $F_{1 \alpha}$ in Eqs. (19) and (21) should be replaced with $\widehat{F}_{1 \alpha}$. 
In summary, for the aforementioned class of forcing schemes, the velocity $\mathbf{u}$ can be used in the equilibrium distribution function $f_{\alpha}^{\mathrm{eq}}$ in Eq. (1), but in the Chapman-Enskog analysis we should ensure that $f_{\alpha}^{(0)}$ in the multiscale expansion (16) is equal to $f_{\alpha}^{\mathrm{eq}}(\rho, \hat{\mathbf{u}})$, which leads to $\sum_{\alpha} \mathbf{e}_{\alpha} f_{\alpha}^{(1)}=-\delta_{t} \mathbf{F}_{1} / 2$. In other words, the multiscale expansion of $f_{\alpha}$ should be based on the actual fluid velocity $\hat{\mathbf{u}}$. The incorrectness of the original analysis lies in the treatment $f_{\alpha}^{(0)}=f_{\alpha}^{\text {eq }}(\rho, \mathbf{u})$ with $\sum_{\alpha} \mathbf{e}_{\alpha} f_{\alpha}^{(1)}=0$. By applying the revised treatment, it can be found that the momentum equation recovered from the aforementioned class of forcing schemes takes the following form:

$$
\begin{aligned}
\partial_{t}(\rho \hat{\mathbf{u}}) & +\nabla \cdot(\rho \hat{\mathbf{u}} \hat{\mathbf{u}}) \\
= & -\nabla p+\nabla \cdot \hat{\boldsymbol{\Pi}}+\mathbf{F} \\
& +\nabla \cdot\left[\left(\tau-\frac{1}{2}\right) \delta_{t}(\hat{\mathbf{u}} \mathbf{F}+\mathbf{F} \hat{\mathbf{u}})-\tau \sum_{\alpha} \mathbf{e}_{\alpha} \mathbf{e}_{\alpha} \hat{F}_{\alpha}\right],
\end{aligned}
$$

where $\hat{\boldsymbol{\Pi}}=\rho v\left[\nabla \hat{\mathbf{u}}+(\nabla \hat{\mathbf{u}})^{\mathrm{T}}\right]$. Obviously, for Wagner's forcing scheme [see Eq. (42)], the macroscopic momentum equation at the Navier-Stokes level can be correctly recovered.

According to Eq. (40) and Eqs. (9) and (11), the following moments can be obtained for the forcing schemes of Kupershtokh et al. and Ladd and Verberg, respectively,

$$
\begin{aligned}
& \sum_{\alpha} \mathbf{e}_{\alpha} \mathbf{e}_{\alpha} \widehat{F}_{\alpha, \mathrm{EDM}} \\
& \quad=\delta_{t}\left(1-\frac{1}{2 \tau}\right)\left[(\mathbf{u F}+\mathbf{F u})+\delta_{t} \frac{\mathbf{F F}}{\rho}\right]+\frac{\delta_{t}^{2}}{4 \tau} \frac{\mathbf{F F}}{\rho}, \\
& \sum_{\alpha} \mathbf{e}_{\alpha} \mathbf{e}_{\alpha} \widehat{F}_{\alpha, \text { Ladd }} \\
& =\delta_{t}\left(1-\frac{1}{2 \tau}\right)\left[(\mathbf{u F}+\mathbf{F u})+\delta_{t} \frac{\mathbf{F F}}{\rho}\right]-\delta_{t}^{2}\left(1-\frac{1}{4 \tau}\right) \frac{\mathbf{F F}}{\rho} .
\end{aligned}
$$

With the above formulations, the actual momentum equations recovered from the forcing schemes of Kupershtokh et al. and Ladd and Verberg can be written as, respectively,

$$
\begin{aligned}
& \partial_{t}(\rho \hat{\mathbf{u}})+\nabla \cdot(\rho \hat{\mathbf{u}} \mathbf{u})=-\nabla p+\nabla \cdot \hat{\boldsymbol{\Pi}}+\mathbf{F}-\delta_{t}^{2} \nabla \cdot\left(\frac{\mathbf{F F}}{4 \rho}\right), \\
& \partial_{t}(\rho \hat{\mathbf{u}})+\nabla \cdot(\rho \hat{\mathbf{u}} \hat{\mathbf{u}}) \\
& \quad=-\nabla p+\nabla \cdot \hat{\boldsymbol{\Pi}}+\mathbf{F}+\delta_{t}^{2} \nabla \cdot\left[\left(\tau-\frac{1}{4}\right) \frac{\mathbf{F F}}{\rho}\right] .
\end{aligned}
$$

The last terms on the right-hand sides of Eqs. (49) and (50) are spatial error terms, which are second order in space since $\delta_{t}=\delta_{x}$ in the standard LB method $\left(c=\delta_{x} / \delta_{t}=1\right)$.

Specifically, Eqs. (35) and (36) involve a time-dependent error term $\partial_{t 2} \mathbf{F}$ and an error term like the viscous term in the momentum equation, whereas there are no such error terms in Eqs. (49) and (50). Moreover, it can be found that the error terms in Eqs. (49) and (50) are proportional to $\nabla \cdot(\mathbf{F F} / \rho)$. Therefore, these terms cannot be neglected when $\nabla \cdot(\mathbf{F F} / \rho)$ is relatively large and are negligible when the force $\mathbf{F}$ is spatially uniform and the variation of density in space is small. Meanwhile, Eqs. (49) and (50) show that the error term (at the Navier-Stokes level) resulting from the forcing scheme of Kupershtokh et al. is not affected by the relaxation time $\tau$, while the error term caused by the forcing scheme of Ladd and Verberg depends on $\tau$. However, according to Eqs. (35) and (36), a contrary conclusion would be drawn. The features of the error terms in Eqs. (49) and (50) will be examined in numerical simulations.

\section{NUMERICAL ANALYSIS}

\section{A. Womersley flow}

Two numerical tests are employed to validate the above theoretical analysis. In simulations, we still use Eqs. (1) and (3) as well as the original forcing terms of the three forcing schemes. First, the Womersley flow bounded by two parallel surfaces is considered, which is an unsteady flow driven by a periodic pressure gradient or a periodic force $F_{x}(t)=$ $-A_{p} \cos (\omega t)$, where $A_{p}$ is the amplitude and $\omega=2 \pi / T$ is the frequency. Obviously, $F_{x}$ is spatially uniform but dependent on time. The analytical solution for the velocity of Womersley flow is given by [15]

$$
U(y, t)=\operatorname{Re}\left(i \frac{A_{p}}{\rho_{0} \omega}\left\{1-\frac{\cosh [(1+i) \sqrt{(\omega / 2 \nu)} y]}{\cosh [(1+i) \sqrt{(\omega / 2 \nu)} L]}\right\} e^{i \omega t}\right)
$$

where $y \in[-L, L]$ with $2 L$ being the channel width, $v$ is the kinematic viscosity, and $\mathrm{Re}$ denotes the real part of the complex number.

The simulations are carried out in a computational domain with $N_{x} \times N_{y}=50 \times 100$. The periodic boundary condition is applied in the $x$ direction and the no-slip boundary condition is imposed at the two solid surfaces (see Sec. III A in Ref. [16]). The period $T$ is set to 1200 , the kinematic viscosity is given by $v=0.1$, and the amplitude $A_{p}$ is taken as 0.0001 . The initial density is chosen as $\rho=\rho_{0}=1$. The simulations begin with $u_{x}=0$ and $u_{y}=0$. Hence $\hat{u}_{x}$ is initially given by $\hat{u}_{x}=$ $0.5 F_{x}(0)$. The numerical results are obtained after running 20 periods.

The numerical results predicted by Wagner's forcing scheme at different times are shown in Fig. 1, from which it can be seen that the numerical results agree well with the analytical solution given by Eq. (51). Quantitatively, the average relative error of the eight cases is about $0.57 \%$. The relative error is defined as $E_{r}=\sum_{y}\left|U-U_{a}\right| / \sum_{y}\left|U_{a}\right|$, in which the subscript $a$ denotes the analytical solution. A comparison of the numerical results has been made between different forcing schemes. In all the cases we find that the numerical results of the three forcing schemes are completely the same as those produced by the forcing scheme of Guo et al. except for a very slight difference at $y^{*}= \pm 0.5$ (the solid walls). By taking the case $t=3 T / 4$ as an example, Table I displays the numerical results obtained by different forcing schemes at $y^{*}=y / 2 L=0,0.15,0.35,0.49$, and 0.5 . For comparison, the analytical results are also listed in the table. The difference at $y^{*}=0.5$ is due to the roundoff errors in numerical computations. 


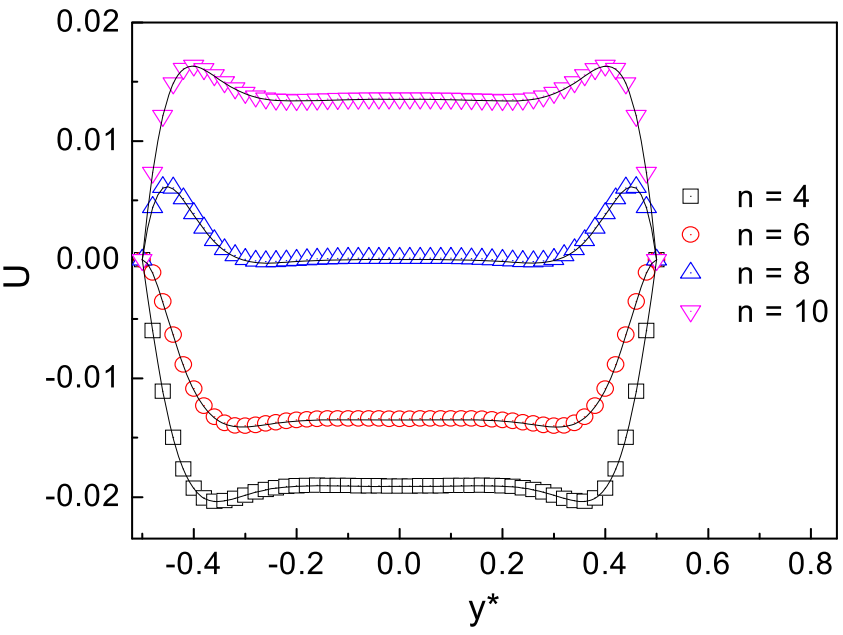

(a)

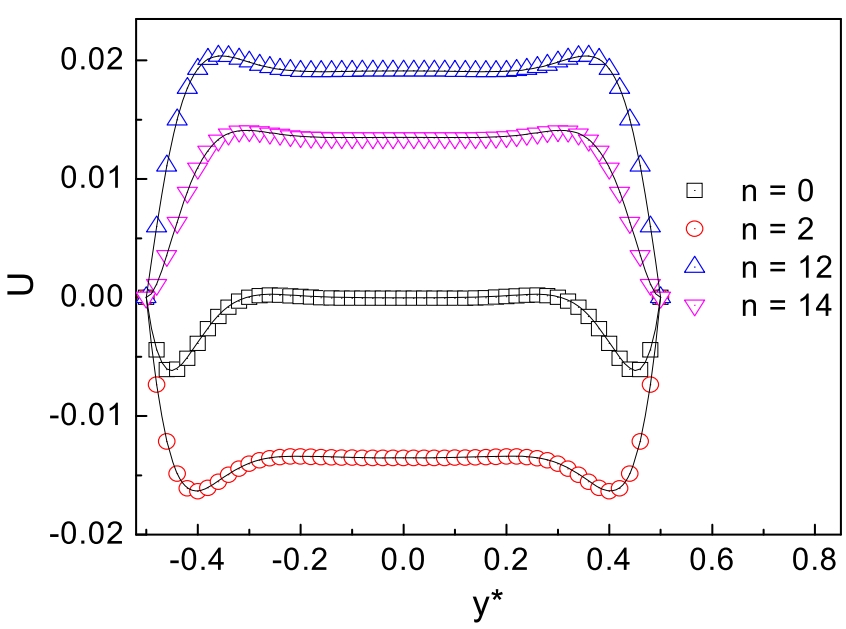

(b)

FIG. 1. Numerical (symbols) and analytical (solid line) results of Womersley flow at different times $t=n T / 16$ with (a) $n=4,6,8,10$ and (b) $n=0,2,12,14$. The numerical results are obtained by Wagner's forcing scheme.

It is clear that if the macroscopic equations recovered from the three forcing schemes satisfy Eq. (32), the time dependence of $F_{x}$ will yield numerical errors according to Eqs. (34)-(36). Correspondingly, their results will be different from the results of the forcing scheme of Guo et al. In contrast, if the macroscopic equations obey Eq. (46), the results of the three forcing schemes should be the same as those of the forcing scheme of Guo et al. since $F_{x}$ is spatially uniform and there are no time-dependent error terms in Eqs. (49) and (50). Obviously, our numerical results confirm the latter.

\section{B. Static flat and circular interfaces}

In this section we consider the simulations of static flat and circular interfaces by the pseudopotential multiphase LB model proposed by Shan and Chen [6,7], which is very sensitive to the forcing scheme and the relaxation time [5]. The pseudopotential interaction force is given by [17]

$$
\mathbf{F}=-G \psi(x) \sum_{\alpha} w_{\alpha} \psi\left(\mathbf{x}+\mathbf{e}_{\alpha} \delta_{t}\right) \mathbf{e}_{\alpha},
$$

where $\psi$ is the pseudopotential, $G$ is the interaction strength, and $w_{\alpha}$ are the weights. For the nearest-neighbor interactions on the D2Q9 lattice, the weights $w_{\alpha}=1 / 3$ for $\left|\mathbf{e}_{\alpha}\right|^{2}=1$ and $w_{\alpha}=1 / 12$ for $\left|\mathbf{e}_{\alpha}\right|^{2}=2$. The pseudopotential is taken as $\psi(\rho)=\psi_{0} \exp \left(-\rho_{0} / \rho\right)$. With such a choice, the thermodynamic consistency can be satisfied when the macroscopic equations are correctly recovered.

The coexistence densities (liquid density $\rho_{L}$ and vapor density $\rho_{V}$ ) are related to $G, \psi_{0}$, and $\rho_{0}$. In the present study we adopt $\psi_{0}=1, \rho_{0}=1$, and $G=-10 / 3$, which leads to $\rho_{L} \approx 2.783$ and $\rho_{V} \approx 0.3675$ [18]. The analytical coexistence densities obey the Maxwell equal-area rule (also called the Maxwell construction). The simulations are carried out in a rectangular domain and the periodic boundary condition is applied in both the $x$ and $y$ directions. For flat interfaces, the lattice system is chosen as $N_{x} \times N_{y}=100 \times 100$ and the initial density is set to $\rho=\rho_{L}$ when $0.25 N_{x} \leqslant x \leqslant 0.75 N_{x}$ and $\rho=\rho_{V}$ when $x>0.75 N_{x}$ or $x<0.25 N_{x}$. The numerical coexistence densities are displayed in Table II, with the relaxation time being examined at $\tau=0.7,1.1$, and 1.5 .

According to Table II, we can see that the numerical coexistence densities obtained by Wagner's forcing scheme are identical to those given by the forcing scheme of Guo et al. Their results are in excellent agreement with the analytical solution of the Maxwell construction $\left(\rho_{L} \approx 2.783\right.$ and $\left.\rho_{V} \approx 0.3675\right)$ and are independent of the relaxation time $\tau$, demonstrating the theoretical analysis in Sec. III. For the forcing scheme of Kupershtokh et al., the numerical results obviously deviate from the analytical solution, although the results are $\tau$-independent. For Ladd and Verberg's forcing scheme, the numerical coexistence densities significantly deviate from the analytical solution and the results vary with $\tau$. Specifically, the deviation increases with the increase of $\tau$. The performances of these two forcing schemes confirm the error terms in Eqs. (49) and (50), i.e., the error term (at

TABLE I. Numerical results obtained by different forcing schemes at $t=3 T / 4$.

\begin{tabular}{lccccc}
\hline \hline Scheme & $y^{*}=0$ & $y^{*}=0.15$ & $y^{*}=0.35$ & $y^{*}=0.49$ & $y^{*}=0.5$ \\
\hline Wagner [12] & 0.0191349 & 0.0190741 & 0.0203897 & 0.00304959 & $4.11605 \times 10^{-19}$ \\
Kupershtokh et al. [13] & 0.0191349 & 0.0190741 & 0.0203897 & 0.00304959 & $4.11605 \times 10^{-19}$ \\
Ladd and Verberg [10] & 0.0191349 & 0.0190741 & 0.0203897 & 0.00304959 & $4.12082 \times 10^{-19}$ \\
Guo [11] & 0.0191349 & 0.0190741 & 0.0203897 & 0.00304959 & $4.10864 \times 10^{-19}$ \\
Eq. (51) & 0.0191013 & 0.0190449 & 0.0203724 & 0.00306534 & 0 \\
\hline \hline
\end{tabular}


TABLE II. Simulation of flat surfaces. Here $\rho_{L}$ and $\rho_{V}$ were obtained by different forcing schemes.

\begin{tabular}{|c|c|c|c|c|c|c|}
\hline \multirow[b]{2}{*}{ Scheme } & \multicolumn{2}{|c|}{$\tau=0.7$} & \multicolumn{2}{|c|}{$\tau=1.1$} & \multicolumn{2}{|c|}{$\tau=1.5$} \\
\hline & $\rho_{L}$ & $\rho_{V}$ & $\rho_{L}$ & $\rho_{V}$ & $\rho_{L}$ & $\rho_{V}$ \\
\hline Guo et al. [11] & 2.785 & 0.369 & 2.785 & 0.369 & 2.785 & 0.369 \\
\hline Wagner [12] & 2.785 & 0.369 & 2.785 & 0.369 & 2.785 & 0.369 \\
\hline Kupershtokh et al. [13] & 2.840 & 0.402 & 2.840 & 0.402 & 2.840 & 0.402 \\
\hline Ladd and Verberg [10] & 2.657 & 0.302 & 2.440 & 0.238 & 2.190 & 0.184 \\
\hline
\end{tabular}

the Navier-Stokes level) resulting from the forcing scheme of Kupershtokh et al. is independent of the relaxation time, while the error term produced by Ladd and Verberg's forcing scheme varies with $\tau$. In the pseudopotential LB model, the coexistence densities are governed by the mechanical stability condition, which is changed when an additional (or error) term is introduced into the macroscopic equations [14].

For circular interfaces, the lattice system is taken as $N_{x} \times N_{y}=200 \times 200$ and a droplet of radius $r=50$ is initially placed in the center of the computational domain. In comparison with flat interfaces, the following two issues should be noted about circular interfaces. First, according to the Laplace law, there is a pressure difference between the liquid and vapor phases in the presence of curved interfaces. Therefore, the Maxwell equal-area rule, which is established when the liquid pressure is equal to the vapor pressure, is theoretically invalid for curved interfaces. Correspondingly, the liquid and vapor densities will deviate from the results obtained by the simulation of flat interfaces, in which $\Delta p=0$. Details about this issue can be found in Refs. [19,20]. Second, the spurious currents in the simulation of flat interfaces are very small. However, for circular interfaces the spurious currents are enlarged by many orders of magnitude, arising from the anisotropy of the high-order terms of the interaction force [21,22]. Meanwhile, with the significant increase of the spurious currents, the velocity-dependent error terms beyond the Navier-Stokes level will also be activated.

Some of the high-order error terms involve the density derivatives and the relaxation time. Accordingly, in the cases of curved interfaces the relaxation time will affect the numerical results even there are no $\tau$-dependent error terms at the NavierStokes level. To suppress such an influence of high-order error terms, a low-density ratio has been adopted in the present study. The numerical coexistence densities obtained from the simulation of circular interfaces are shown in Table III.

TABLE III. Simulation of circular interfaces. Here $\rho_{L}$ and $\rho_{V}$ were obtained by different forcing schemes.

\begin{tabular}{|c|c|c|c|c|c|c|}
\hline \multirow[b]{2}{*}{ Scheme } & \multicolumn{2}{|c|}{$\tau=0.7$} & \multicolumn{2}{|c|}{$\tau=1.1$} & \multicolumn{2}{|c|}{$\tau=1.5$} \\
\hline & $\rho_{L}$ & $\rho_{V}$ & $\rho_{L}$ & $\rho_{V}$ & $\rho_{L}$ & $\rho_{V}$ \\
\hline Guo et al. [11] & 2.793 & 0.366 & 2.800 & 0.371 & 2.806 & 0.374 \\
\hline Wagner [12] & 2.793 & 0.366 & 2.800 & 0.371 & 2.806 & 0.374 \\
\hline Kupershtokh et al. [13] & 2.856 & 0.403 & 2.862 & 0.406 & 2.864 & 0.408 \\
\hline Ladd and Verberg [10] & 2.627 & 0.297 & 2.480 & 0.250 & 2.320 & 0.209 \\
\hline
\end{tabular}

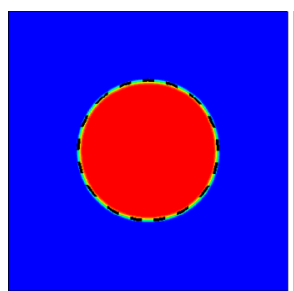

(a)

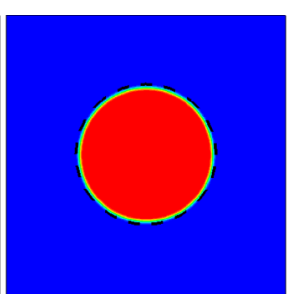

(b)

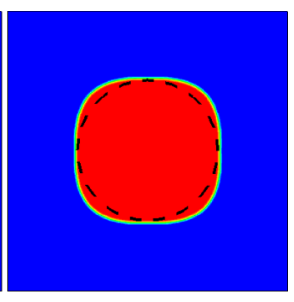

(c)
FIG. 2. Simulation of circular interfaces: steady-state density contours $(\tau=0.7)$ obtained by the forcing schemes proposed by (a) Wagner [12], (b) Kupershtokh et al. [13], and (c) Ladd [10].

Obviously, the forcing schemes of Wagner and Guo et al. still yield the same results. Nevertheless, the results are different from those in Table II due to the influence of the pressure difference (the Laplace law). Moreover, a slight variation with $\tau$ can be observed in the results. Similarly, the results of the forcing scheme of Kupershtokh et al. also vary slightly with the relaxation time. The reason has just been illustrated. With the increase of the density ratio, the variations of $\rho_{L}$ and $\rho_{V}$ with $\tau$ (or the viscosity) will be more severe. An alternative approach is using the multi-relaxation-time collision operator [23-25] to reduce the variations caused by the relaxation times since most of the relaxation times can be fixed when varying the viscosity.

Finally, the steady-state density contours obtained by different forcing schemes are compared in Fig. 2. The dashed circle denotes the theoretical location of the interface (the radius $r=50$ ). Obviously, Wagner's forcing scheme performs best among the three forcing schemes. Meanwhile, it can be seen that the droplet radius predicted by the forcing scheme of Kupershtokh et al. is a little smaller than $r=50$ and the droplet produced by Ladd and Verberg's forcing scheme is noncircular, which shows the influence of the error term on the size and shape of the droplet.

\section{SUMMARY}

We have clarified a critical issue about the ChapmanEnskog analysis for a class of forcing schemes in the LB method, in which the velocity in the equilibrium density distribution function is given by $\mathbf{u}=\sum_{\alpha} \mathbf{e}_{\alpha} f_{\alpha} / \rho$, but the actual fluid velocity is defined as $\hat{\mathbf{u}}=\mathbf{u}+\delta_{t} \mathbf{F} /(2 \rho)$. In the available Chapman-Enskog analysis of this class of forcing scheme, $f_{\alpha}^{(0)}$ in Eq. (18) was taken as $f_{\alpha}^{\mathrm{eq}}(\rho, \mathbf{u})$. In the present work, we demonstrated that the usual Chapman-Enskog analysis for this class of forcing schemes should be revised in order to derive the actual macroscopic equations recovered from these forcing schemes.

It was shown theoretically and numerically that the forcing schemes of Wagner and Guo et al. are equivalent at the NavierStokes level, which confirms the consistency between the Chapman-Enskog analysis and the Taylor expansion analysis. Using the revised Chapman-Enskog analysis, the forcing schemes proposed by Ladd and Verberg [10] and Kupershtokh et al. [13] have also been analyzed. It is believed that the present analysis is useful for clarifying some inconsistencies 
in the literature and we hope that this study would be helpful for general readers to better understand the forcing schemes in the LB community. Finally, it is worth mentioning that the Chapman-Enskog analysis for the forcing scheme proposed by Shan et al. [7,26] should be conducted in a similar way, in which the velocity in the equilibrium distribution function is give by $\mathbf{u}^{\mathrm{eq}}=\mathbf{u}+\tau \delta_{t} \mathbf{F} / \rho$ and the actual fluid velocity is the same as above [26].

\section{ACKNOWLEDGMENTS}

This work was financially supported by the National Natural Science Foundation of China (Grant No. 51506227), the Foundation for the Author of National Excellent Doctoral Dissertation of China (Grant No. 201439), and the Los Alamos National Laboratory's Lab Directed Research \& Development Program.
[1] S. Chen and G. D. Doolen, Annu. Rev. Fluid Mech. 30, 329 (1998).

[2] C. K. Aidun and J. R. Clausen, Annu. Rev. Fluid Mech. 42, 439 (2010).

[3] S. Succi, The Lattice Boltzmann Equation for Fluid Dynamics and Beyond (Oxford University Press, Oxford, 2001).

[4] Z. Guo and C. Shu, Lattice Boltzmann Method and Its Applications in Engineering (World Scientific, Singapore, 2013).

[5] Q. Li, K. H. Luo, Q. J. Kang, Y. L. He, Q. Chen, and Q. Liu, Prog. Energy Combust. Sci. 52, 62 (2016).

[6] X. Shan and H. Chen, Phys. Rev. E 47, 1815 (1993).

[7] X. Shan and H. Chen, Phys. Rev. E 49, 2941 (1994).

[8] X. He, X. Shan, and G. D. Doolen, Phys. Rev. E 57, R13 (1998).

[9] J. M. Buick and C. A. Greated, Phys. Rev. E 61, 5307 (2000).

[10] A. J. C. Ladd and R. Verberg, J. Stat. Phys. 104, 1191 (2001).

[11] Z. Guo, C. Zheng, and B. Shi, Phys. Rev. E 65, 046308 (2002).

[12] A. J. Wagner, Phys. Rev. E 74, 056703 (2006).

[13] A. L. Kupershtokh, D. A. Medvedev, and D. I. Karpov, Comput. Math. Appl. 58, 965 (2009).
[14] Q. Li, K. H. Luo, and X. J. Li, Phys. Rev. E 86, 016709 (2012).

[15] I. G. Currie, Fundamental Mechanics of Fluids (CRC, Boca Raton, 2012).

[16] Q. Li, K. H. Luo, Q. J. Kang, and Q. Chen, Phys. Rev. E 90, 053301 (2014).

[17] X. Shan, Phys. Rev. E 77, 066702 (2008).

[18] Z. Yu and L.-S. Fan, J. Comput. Phys. 228, 6456 (2009).

[19] E. S. Kikkinides, A. G. Yiotis, M. E. Kainourgiakis, and A. K. Stubos, Phys. Rev. E 78, 036702 (2008).

[20] Q. Li and K. H. Luo, Appl. Therm. Eng. 72, 56 (2014).

[21] X. Shan, Phys. Rev. E 73, 047701 (2006).

[22] M. Sbragaglia, R. Benzi, L. Biferale, S. Succi, K. Sugiyama, and F. Toschi, Phys. Rev. E 75, 026702 (2007).

[23] P. Lallemand and L.-S. Luo, Phys. Rev. E 61, 6546 (2000).

[24] Q. Li, K. H. Luo, and X. J. Li, Phys. Rev. E 87, 053301 (2013).

[25] A. Xu, T. S. Zhao, L. An, and L. Shi, Int. J. Heat Fluid Flow 56, 261 (2015).

[26] X. Shan and G. Doolen, J. Stat. Phys. 81, 379 (1995). 